\title{
Simulación de Redes de Sensores Inalámbricos: un Modelo Energético a Nivel de Nodo-Sensor Bajo LAS ESPECIFICACIONES IEEE 802.15.4 ${ }^{\mathrm{TM}}$ Y ZiGBEE
}

\author{
Simulation of Wireless Sensor Networks: an Energy \\ Model at leVel of Sensor-nOde Under the IeEe SpeCifications \\ AND ZIGBEE 802.15.4TM
}

\section{${ }^{1}$ Milton Osvaldo Amarillo Rojas, ${ }^{2}$ Luis Carlos Trujillo Arboleda}

\author{
Pontificia Universidad Javeriana, Departamento de Electrónica, Bogotá, D.C., Colombia \\ ${ }^{1}$ mamarillo@javeriana.edu.co ${ }^{2}$ trujillo.luis@javeriana.edu.co
}

Recibido: 25/05/2014 • Aprobado: 22/05/2014

\section{RESUMEN}

Este artículo presenta el desarrollo de un modelo de simulación para una red de sensores inalámbricos que se rige por las especificaciones IEEE 802.15.4 y ZigBee y que utiliza la configuración de la beacon habilitada. El modelo de simulación también implementa un modelo de consumo de energía para los tres tipos de nodossensores de la red (Coordinador ZigBee, Router y Dispositivo Final). El principal aporte de este trabajo radica en que por medio de la simulación se puede estimar el consumo de energía de un nodo-sensor y, de esta forma, analizar el consumo de energía de cada uno de los tipos del mismo. Así mismo, al poder estimar el consumo de energía del nodo-sensor, también se puede estimar su tiempo de vida. Por último, se analiza la razón, por la cual, el consumo de energía de los nodos es inversamente proporcional a la latencia de los datos dentro de la red.

Palabras clave: bajo consumo, beacon, consumo de energía, coordinador, dispositivo final, IEEE 802.15.4, latencia, OPNET, router, simulación, WPAN, WSN, ZigBee.

\section{Abstract}

This paper shows the development of a simulation model for a wireless sensor network in accordance with IEEE 802.15.4 and ZigBee standards, and it uses the beacon-enabled configuration. The simulation model also implements a model of the energy consumption for the three types of nodes-sensors (ZigBee Coordinator, ZigBee Router y End Device). The main contribution of this work is that through simulation is possible to estimate the energy consumption of a node-sensor, and so in this way is possible to analyze the energy consumption of each of the type node-sensors, furthermore to be able to estimate energy consumption of the node-sensor, also is possible estimate the time of life of the node-sensor. Finally, it is analyzed the reason why, the energy consumption of the nodes is inversely proportional to data latency within the network.

Keywords: beacon, coordinator, end device, energy consumption, IEEE 802.15.4, latency, low consumption, OPNET, router, simulation, WPAN, WSN, ZigBee. 


\section{INTRODUCCIÓN}

Las redes de sensores inalámbricos (WSN) se utilizan en hogares inteligentes [1], automatización industrial [1], agricultura [2], etc.

Las principales características y ventajas de los dispositivos (nodos-sensores) de redes de sensores inalámbricos que utilizan las especificaciones IEEE 802.15.4/ZigBee son el bajo costo del hardware y el bajo consumo de energía, para aplicaciones que no necesitan un alto flujo de datos.

El bajo consumo de energía es importante, porque el tiempo de vida de una red de sensores inalámbricos está ligado directamente al consumo de energía de sus nodos-sensores, debido a que la energía disponible en la batería de un nodo-sensor es un recurso agotable y en la mayoría de los casos no renovable (baterías alcalinas). Por tanto, es importante el estudio de la dinámica del consumo de la energía en los nodos-sensores, con el fin de buscar una gestión óptima del recurso energético [3].

En el estudio de redes de sensores inalámbricos es común usar modelos de simulación, ya que estos brindan la posibilidad de montar múltiples escenarios (diferentes configuraciones de red) con múltiples configuraciones de sus nodossensores (diferentes atributos), lo cual es muy difícil en un ambiente real. Además, los modelos de simulación de redes de sensores inalámbricos brindan una ventaja adicional que es el poder analizar en tan solo unos minutos $u$ horas el comportamiento que tendría una WSN en un periodo de tiempo muy largo (e.g. 2 o 3 años), lo cual también es muy difícil en un ambiente real [3].

En este artículo se describe un modelo de simulación de redes de sensores inalámbricos que se basa en las especificaciones IEEE 802.15.4 [6] y ZigBee [4]. El modelo de simulación tiene tres modelos de nodo (coordinador, enrutador y dispositivo final), cada uno de los cuales consta de un modelo de flujo de datos y un modelo energético. Con el modelo energético se estima el consumo de energía de cada uno de los nodos-sensores que participan en la red y con el modelo de flujo de datos se hace la estimación del retardo que sufren los datos desde el origen hasta el destino (latencia) y se determina el tráfico enviado y el tráfico recibido durante el transcurso de la simulación [3].

\section{Descripción General IeEe 802.15.4/ZIGBEE}

\section{Nodos de red IEEE 802.15.4/ZigBee}

Cada uno de los nodos de red representa un sensor inalámbrico, el cual basa su configuración y demás en el estándar descrito en la norma ZigBee del 2007, la cual contempla tres tipos de nodos de red: dispositivo final, coordinador PAN y enrutador [4]. Es preciso mencionar que tanto la capa física como la capa MAC se han construido de tal manera que cumplan con las especificaciones impuestas por el estándar IEEE 802.15.4-2003 [6].

1) Dispositivo final. Es un dispositivo de la norma 802.15.4-2003 con funciones reducidas (RFD) o completas (FFD), y que no es un coordinador ZigBee ni tampoco un enrutador ZigBee. Este tipo de nodo tiene las siguientes características [5]:

- Componente de red opcional.

- No permite asociación.

- No participa en el enrutamiento.

- Puede ser un dispositivo con funciones reducidas (RFD) e implementa un subconjunto reducido de la pila de protocolos.

2) Coordinador ZigBee. Es un coordinador PAN según la norma 802.15.4-2003, el cual trabaja en la capa de red del protocolo ZigBee. Sus características principales son [5]: 
- Uno para cada red ZigBee.

- Inicia y configura la formación de la red.

- Es un coordinador de red de área personal de la norma IEEE 802.15.4.

- Es el enrutador cuando la red está formada.

- Es un dispositivo FFD (Full Funcional Device), el cual implementa toda la pila de protocolos.

3) Enrutador. Es un dispositivo confunciones completas (FFD) según la norma 802.15.4-2003, el cual, aunque no es un coordinador ZigBee, puede actuar como un coordinador PAN de la norma 802.15.4-2003 dentro de su espacio de operación. Además, este dispositivo es capaz de enrutar mensajes entre dispositivos y asociar dispositivos tanto en su capa MAC como en su capa de red. Sus principales características son [5]:

- Componente opcional de red.
- Se asocia con el coordinador ZigBee (ZC) o con un router ZigBee previamente asociado (ZR).

- Puede ser un coordinador PAN según la norma IEEE 802.15.4.

- Participa en el enrutamiento multi-salto de los mensajes.

- Es un dispositivo FFD (Full functional device), el cual implementa toda la pila de protocolos.

\section{4) Interacción entre las normas IEEE}

802.15.4/ZigBee. Para que se dé un correcto funcionamiento entre las distintas capas de los protocolos que han de implementarse en un nodo sensor inalámbrico compatible con las normas IEEE 802.15.4 (Física y MAC) y ZigBee (red, aplication suport y aplicación), ha de ser tenido en cuenta el formato general de trama de cada una de las capas de la pila de protocolos IEEE 802.15.4 y ZigBee, el cual se presenta en la Fig. 1.

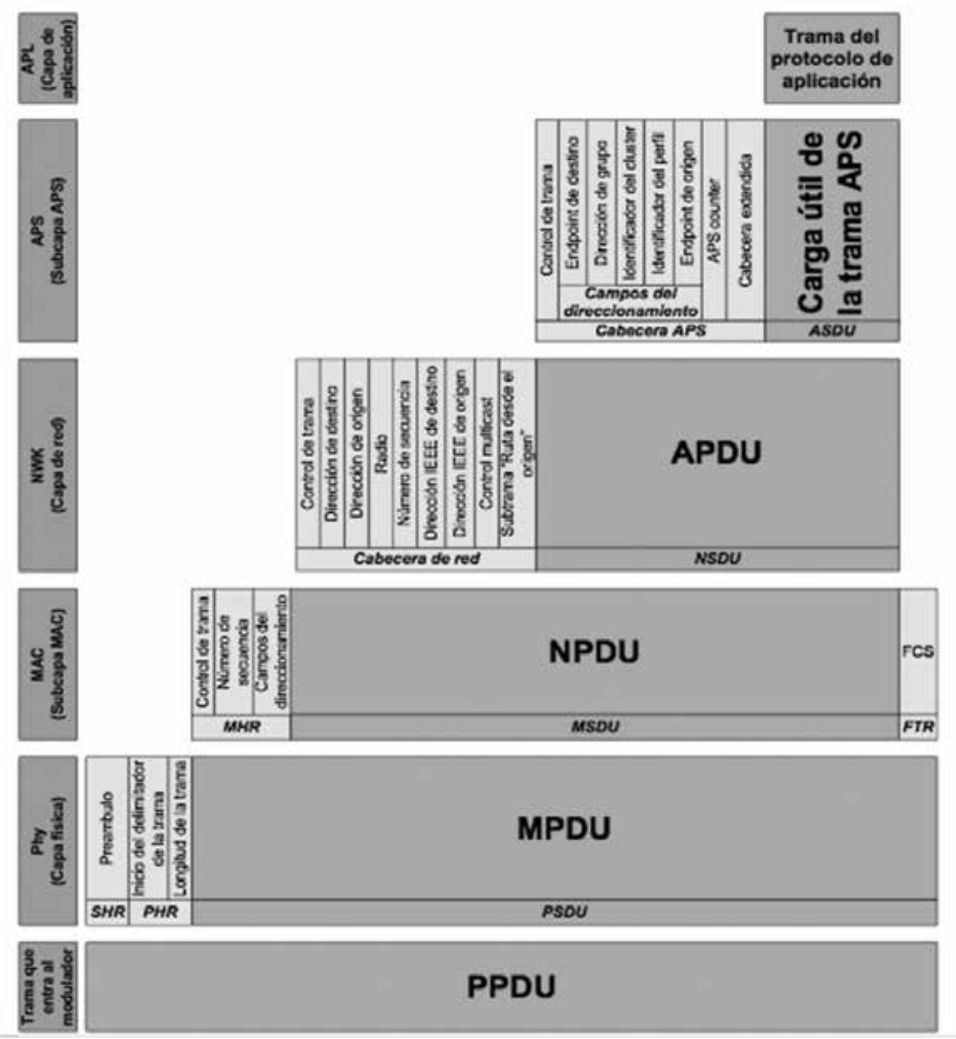

Fig. 1 Formato general de las tramas de cada una de las capas de las especificaciones IEEE 802.15.4/ZigBee [6][4]. 


\section{DESCRIPCION DEL Modelo de Simulación}

\section{A. Descripción General}

El trabajo consiste en el diseño e implementación de un modelo de simulación para una red de sensores inalámbricos que se rige por las especificaciones IEEE 802.15.4 [6] y ZigBee [4]. El modelo de simulación modela el flujo de datos y el comportamiento energético (consumo de energía) de los nodos-sensores que conforman una red que utiliza las especificaciones IEEE 802.15.4/ ZigBee. Para el desarrollo del modelo de simulación se utilizaron tres dominios de modelamiento, los cuales se organizan jerárquicamente en modelo de red, modelo de nodo y modelo de procesos, tal como se muestra en la Fig. 2 [3].

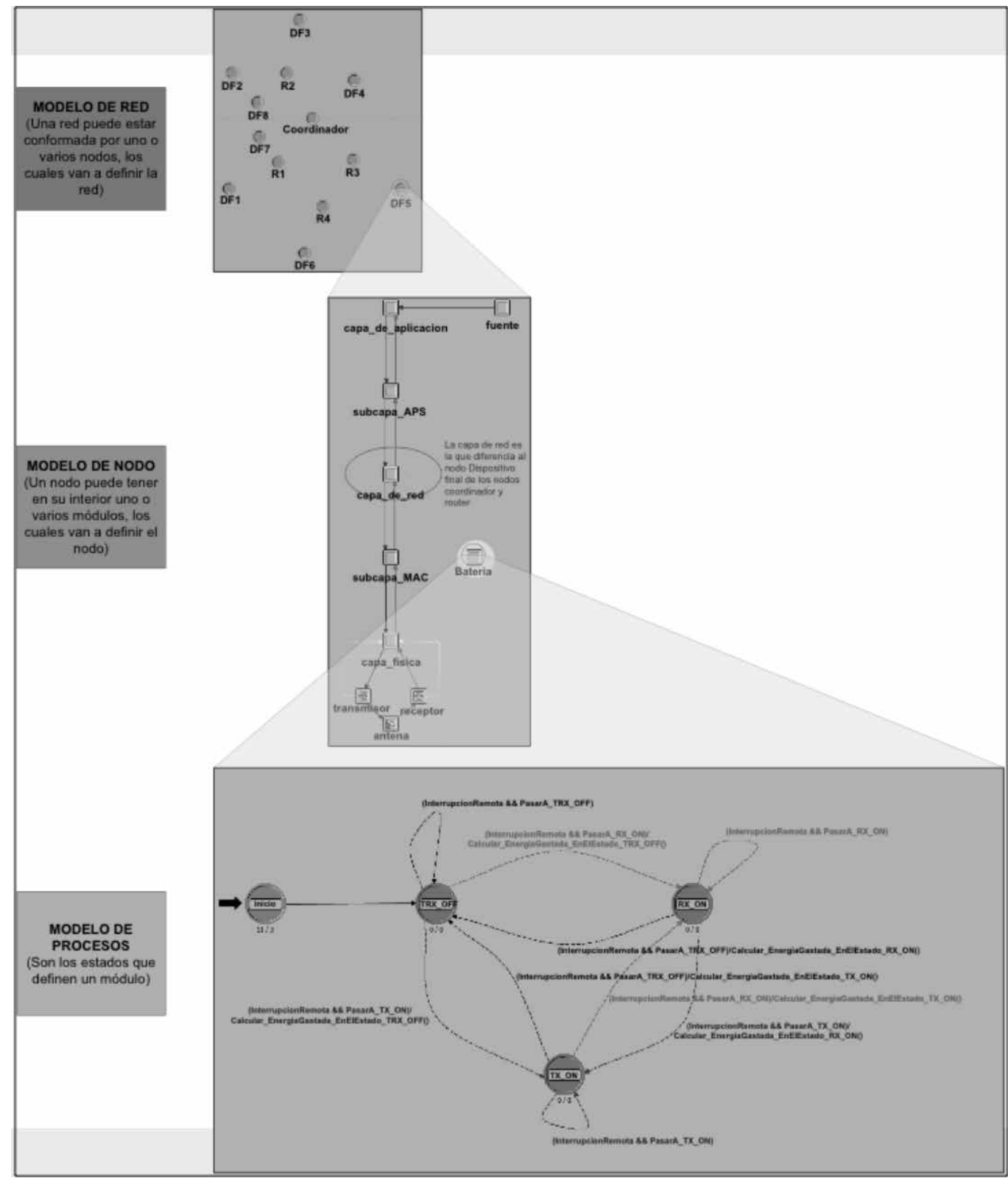

Fig. 2 Jerarquía utilizada para el desarrollo del modelo de simulación [3] 


\section{B. Modelo de Simulación en el Dominio de Red (Modelo de Red)}

La topología de red de sensores inalámbricos que se implementó se encuentra compuesta por ocho dispositivos finales ZigBee (DF), cuatro routers ZigBee (R) y un coordinador ZigBee (coordinador), con sus respectivas direcciones de red, tal como se muestra en la Fig. 3 [3].

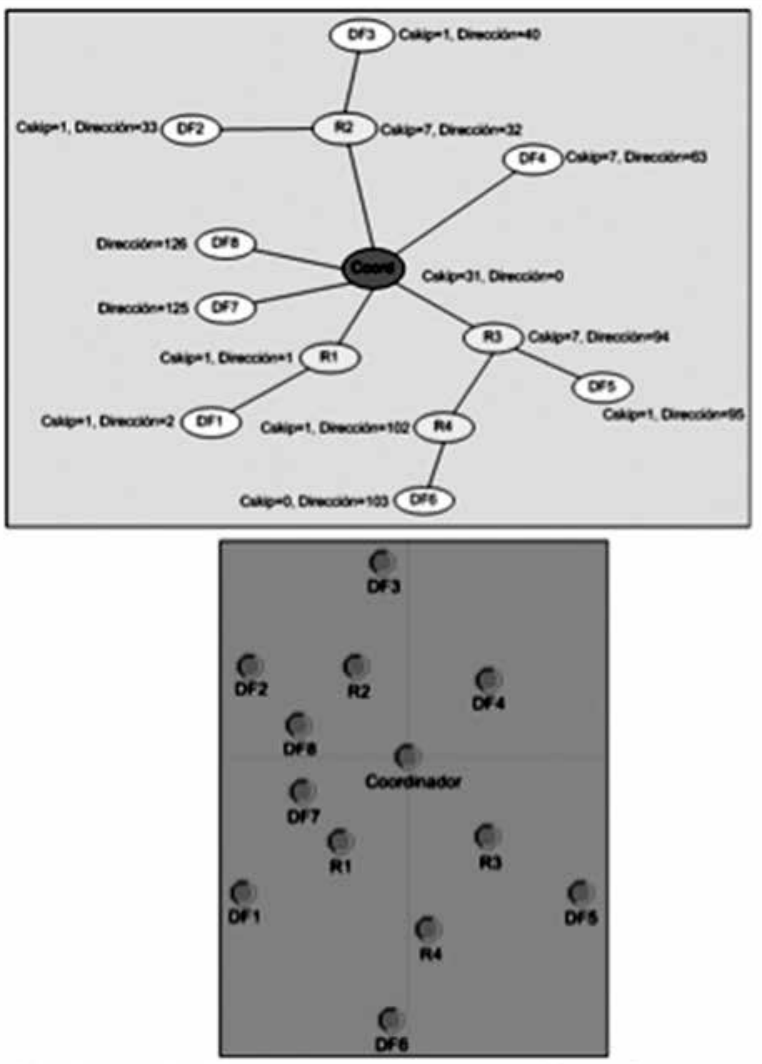

Fig. 3 Asociación de nodos en topología de árbol

En la Fig. 3 en la parte superior e observa la asociación que existe entre los nodos dentro de una topología de árbol y abajo se observa la misma topología de red pero montada sobre OPNET [4[, [3].

\section{Modelo de Simulación en el Dominio deNodo (Modelo de Nodo)}

En el dominio de nodo se desarrollaron tres tipos de nodo (coordinador, router y dispositivo final), los cuales servirán para construir el modelo de red IEEE 802.15.4/ZigBee [6][4], tal como se puede observar en la Fig. 4 [3].

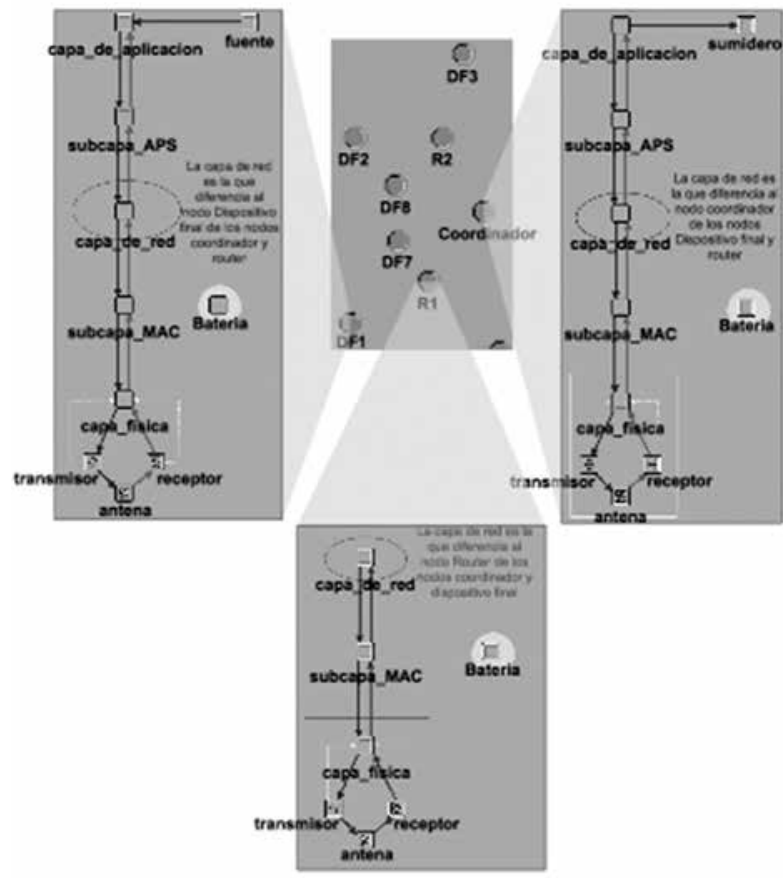

Fig. 4 Modelo de nodo para el dispositivo final izquierda), el router (centro) y el coordinador (derecha) [3].

Al lado izquierdo de la Fig. 4 se observa el modelo de nodo del dispositivo final, el cual está compuesto por 10 módulos, 13 packet streams y 2 statistic wires. En el centro de la Fig. 4 se observa el modelo de nodo del router, el cual está compuesto por 7 módulos, 8 packet streems y 2 statistics wires. Al lado derecho de la Fig. 4 se observa el modelo de nodo del coordinador ZigBee, el cual está compuesto por 10 módulos, 13 packet streems y 2 statistic wires [3].

El modelo de la capa física, la subcapa de enlace de datos y el consumo de energía del modelo de nodo de los tres tipos de nodos-sensores se modeló de la misma forma. La capa física está compuesta por un módulo antena, un módulo transmisor, un módulo receptor y un módulo de procesos llamado capa física. La subcapa de enlace de datos está compuesta por un módulo llamado subcapa MAC. El modelo de consumo de energía se implementó en el módulo llamado Batería. El modelo de la capa de red es diferente en el modelo de nodo de los tres tipos de 
nodo-sensores, ya que es en esta capa en la que se diferencian los tres tipos de nodos-sensores, debido a las funciones que deben desempeñar cada uno de los tres tipos de nodosensores [3].

Como se puede observar en la Fig. 4, el modelo de nodo del router solo tiene hasta capa de red, ya que el router únicamente se dedica a retransmitir los datos en la red y no necesita (en este modelo) procesar los datos de las capas superiores (capas de transporte y aplicación) [3].

\section{Modelo de simulación en el dominio de Proceso (Modelo de Proceso).}

Cada módulo tiene asociado un único modelo de proceso, debido a que este contiene la lógica que debe seguir cada uno de los módulos; es decir, el modelo de proceso determina el comportamiento del módulo: por ejemplo, el módulo de la subcapa MAC tiene asociado un modelo de proceso que define el comportamiento que se debe tener en cuenta en este módulo. Este modelo de proceso de la subcapa MAC se construyó según la especificación de la subcapa MAC del estándar IEEE 802.15.4 [6].

\section{Metodología Utilizadapara el Desarrollo del Modelo DE SimULACIÓN [3]}

Para el desarrollo del modelo de simulación se siguió una metodología iterativa, con el fin de darle un buen soporte, la cual se muestra a manera de diagrama de flujo en la Fig. 5. En el desarrollo de un modelo de simulación no es suficiente con la construcción del código; es necesario verificarlo y validarlo para darle un buen grado de confiabilidad, mientras que la calibración busca optimizarlo para incrementar el grado de confiabilidad. Esta metodología se explica a continuación [3]:

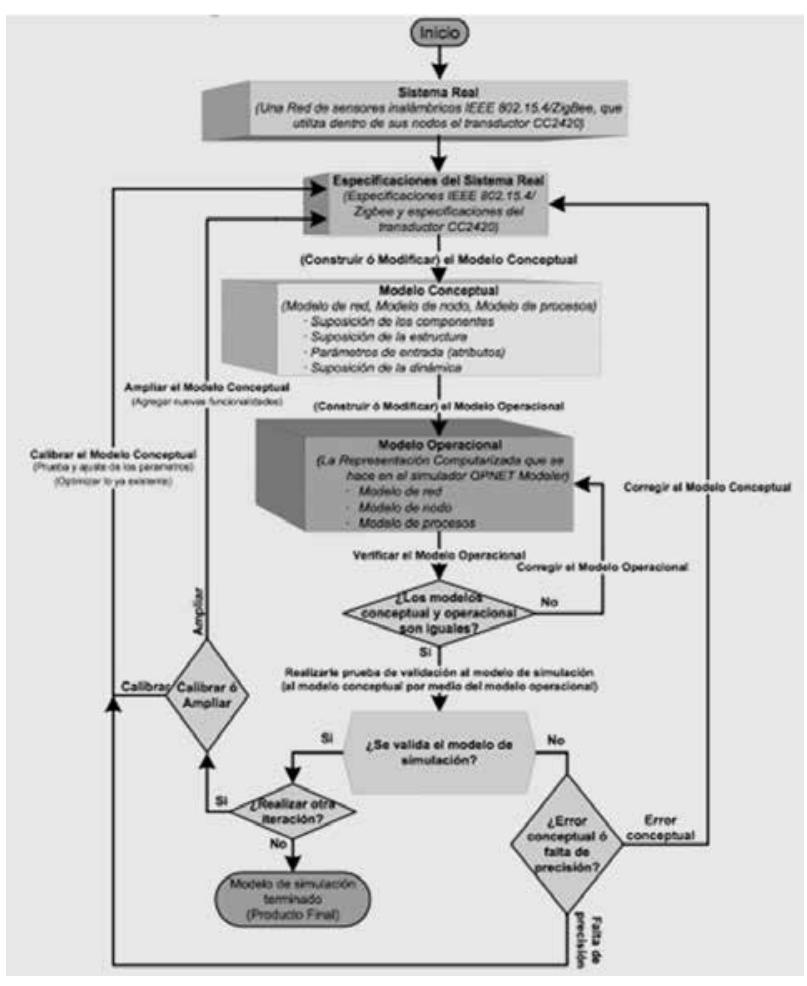

Fig. 5 Modelo de nodo para el Dispositivo Final (izquierda), el Router (centro) y el Coordinador (derecha) [3]

\section{A. Primera Iteración [3]}

1) Sistema real: para el modelo de simulación de la WSN se tuvieron en cuenta dispositivos del mundo real que implementaban las especificaciones IEEE 802.15.4/ZigBee [6][4], tal como el TELOSB [7] y el Micaz [8], los cuales tienen incorporado dentro de su arquitectura de hardware al transductor CC2420 [9] que implementa la especificación IEEE 802.15.4 [6].

2) Especificaciones del sistema real: al no tener físicamente los dispositivos del mundo real, tal como el TELOSB [7], el MICAZ [8] y el CC2420 [9], se procedió a buscar las especificaciones de dichos dispositivos y empezar a trabajar el modelo conceptual a partir de las hojas de especificaciones de los mismos; es decir, el modelo conceptual se construyó a partir de las especificaciones IEEE 802.15.4/ZigBee [6][4] y de las especificaciones de los fabricantes de los dispositivos TELOSB [7], el MICAZ [8] y el CC2420 [9]. 
3) Modelo Conceptual: para la construcción del modelo conceptual se tuvo en cuenta la arquitectura y el funcionamiento del simulador de redes que se iba a utilizar: el OPNET [10]; además, se tuvieron en cuenta las especificaciones IEEE 802.15.4/ZigBee [6], [4] y las especificaciones del transductor CC2420 [9].

4) Modelo operacional: se utilizó el modelo conceptual (todavía no depurado) para la construcción del modelo operacional (modelo computacional) el cual se construyó en el simulador de redes OPNET [10]. Tanto en el modelo conceptual como en el modelo operacional, se utilizaron tres tipos de modelo para una WSN, los cuales se organizan jerárquicamente en modelo de red, modelo de nodo y modelo de procesos. La razón por la que los modelos sean iguales, tiene que ver con el hecho de que el modelo operacional (computacional) debe ser una copia fiel del modelo conceptual y esto se debe ver reflejado positivamente en el proceso de verificación del modelo operacional (computacional).

5) Verificar el modelo operacional: es el proceso mediante el cual el modelo operacional es revisado minuciosamente (desde el código y las impresiones "printf y op_pk_print()" que quedan en la consola de simulación), con el fin de que represente fielmente al modelo conceptual.

6) Validar el modelo de simulación: el modelo conceptual se valida a través del modelo operacional, ya que en el modelo operacional que se construyó en OPNET [10] es donde se pueden recolectar las estadísticas de la simulación, para luego ser analizadas y así determinar cualitativamente (en este caso) si la WSN tiene un comportamiento lógico y qué se esperaría de la red. Adicionalmente, este análisis lógico de la red puede ser apoyado por un log para determinar cualitativamente si la red se está comportando como se debería comportar (según las especificaciones).
B. Iteraciones que se realizan (segunda, tercera, cuarta, etc.), y criterio para la terminación del modelo [3]

Cada vez que se realiza una iteración se busca mejorar el modelo de simulación anterior y para lograr mejorar el modelo de simulación hay que hacer modificaciones tal como se muestra en la Fig. 5.

1) Especificaciones del sistema real: el comienzo de cada iteración, inicia en la revisión de las especificaciones del sistema real, para luego, si es el caso, modificar el modelo conceptual.

2) Modelo conceptual: el modelo conceptual se modifica teniendo la revisión de las especificaciones del sistema real. Si el modelo conceptual se modifica, quiere decir que el modelo operacional ya no representará al modelo conceptual

3) Modelo operacional: el modelo operacional será modificado si el modelo conceptual se modificó; entonces, se volverá a proceder a realizar la verificación del modelo operacional, para que este represente fielmente el modelo conceptual. La validación del modelo se realiza de igual forma a como se realiza en la primera iteración (ya mencionado anteriormente).

\section{4) Criterio para la terminación del modelo:} la Fig. 6 es una buena referencia para decidir cuándo terminar el modelo de simulación, ya que involucra dos aspectos claves para el desarrollador y el usuario del modelo: costo para realizar el modelo (tiempo y dinero) y valor del modelo para el usuario [11]. Se puede observar que a medida que se quiera obtener una mayor confianza (que se aproxime al 100\%) el costo de construcción (calibración) aumentará cada vez más rápido mientras que el valor del modelo crecerá cada vez más despacio. El criterio de terminación del modelo en este caso se tomó partiendo del costo en tiempo para el desarrollador, con el fin de entregar un producto con un buen valor para el usuario (el Departamento de Ingeniería Electrónica). 


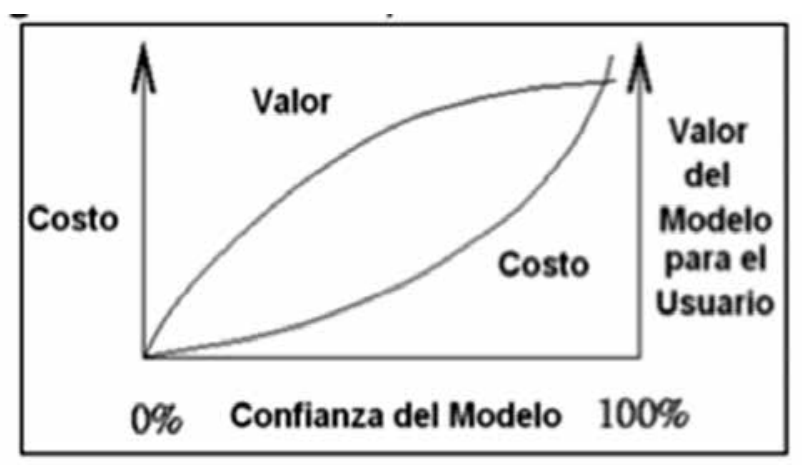

Fig. 6 Valor, costo y confianza del modelo [11]

\section{Resultados}

El consumo de energía de la Fig. 7 corresponde a la topología de red de la Fig. 3 y se observa que el consumo de energía de los routers (color amarillo y cian, Fig. 7) es mucho mayor que el consumo de energía del coordinador ZigBee El consumo de energía de la Fig. 7 corresponde a la topología de red de la Fig. 3 y se observa que el consumo de energía de los routers (color amarillo (5) y cian (4), Fig. 7) es mucho mayor que el consumo de energía del coordinador ZigBee (color azul (1), Fig. 7) y de los dispositivos finales (color rojo (2) y verde (3), Fig. 7), los routers tienen el doble de periodos activos (líneas con mayor pendiente) que los dispositivos finales y el coordinador ZigBee. Es importante entender que en el zoom, la línea de mayor pendiente representa a los periodos activos (TX_ON o RX_ON) y la de menor pendiente representa a los periodos inactivos (transmisor y receptor apagados [TRX_OFF]) [3].

Los nodos sensores de tipo router gastan mucha más energía (más o menos el doble [en este modelo lineal]) que los nodos sensores de tipo coordinador ZigBee o dispositivo final, debido al doble papel que deben cumplir los nodos sensores de tipo router (dentro de la red ZigBee [4]) para poder enrutar los datos, ya que durante un periodo de supertrama deben comportarse como dispositivos asociados a un coordinador PAN y durante otro periodo de tiempo deben comportarse como coordinadores PAN [3].

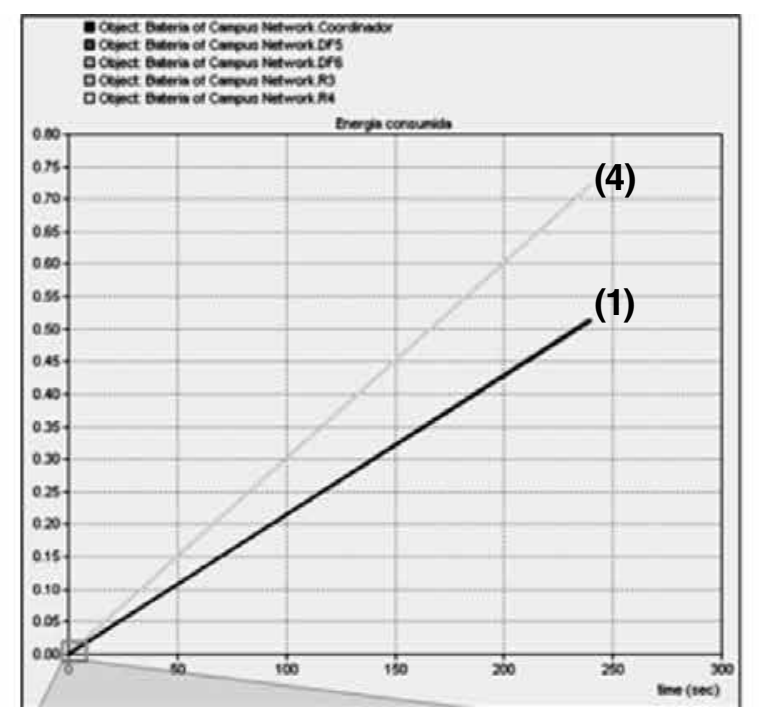

(4)
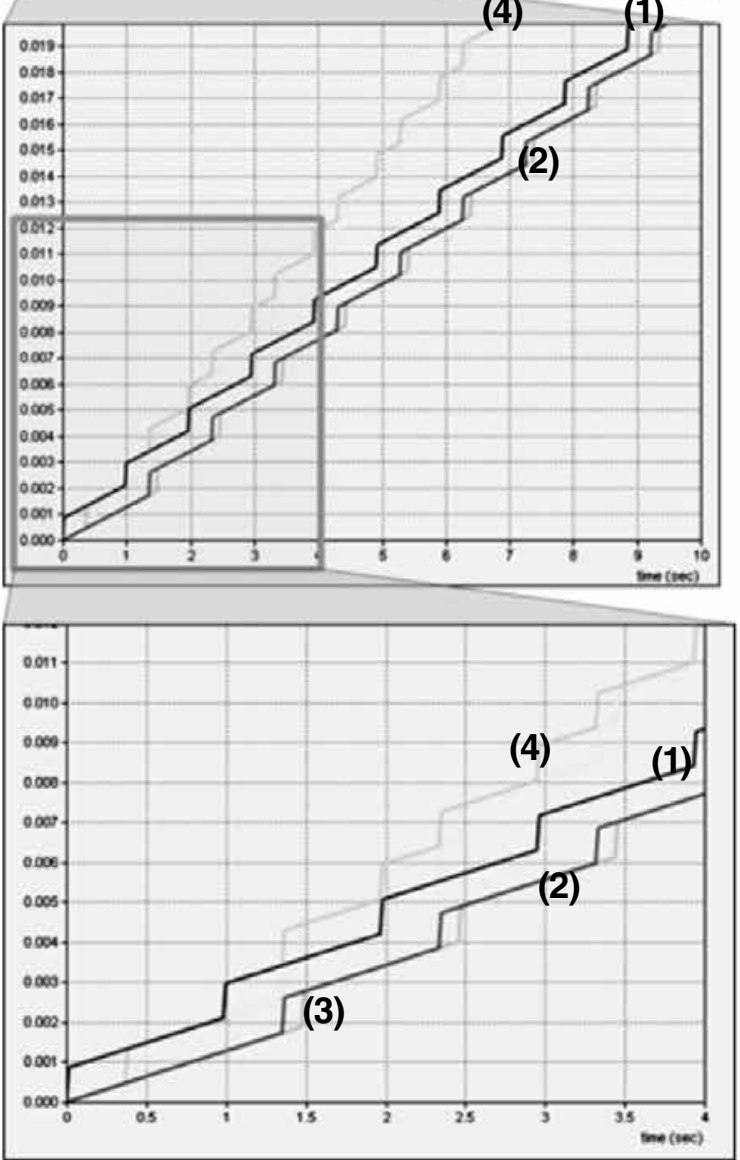

Fig. 7 Consumo de energía del coordinador, R3, R4 DF5 y DF6, de la topología de red de la Fig. 3. [3]

De lo anterior se concluye que durante un intervalo específico de tiempo (intervalo de la beacon del coordinador ZigBee), el coordinador ZigBee y los dispositivos finales, solo estarán activos durante una duración de supertrama, mientras que 
el router estará activo durante dos periodos de supertrama: una duración de supertrama cuando actúa como dispositivo asociado a un coordinador PAN, y otra duración de supertrama cuando actúa como coordinador de una PAN, lo que se puede ver reflejado en las pendientes que tienen mayor inclinación, ya que son el doble de las del coordinador, y los dispositivos finales, tal como se muestra en las gráficas de la Fig. 7 y la Fig. 8 [3].
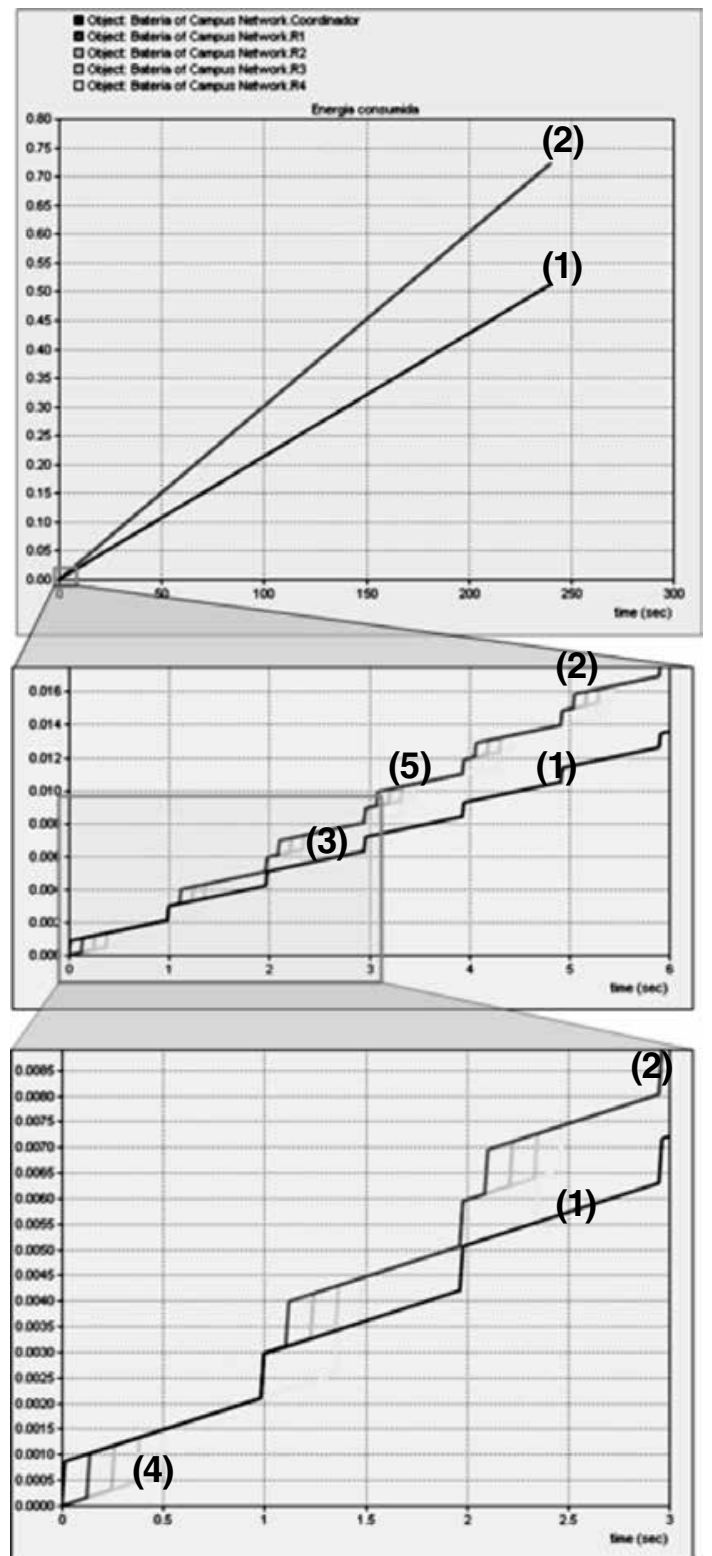

Fig. 8 Consumo de energía de los dispositivos que emiten trama beacon (coordinador y routers) de la topología de red de la Fig. 3 [3]
Todos los dispositivos finales (fuentes) están enviándole datos al coordinador ZigBee (sumidero) y estos, a su vez, no están recibiendo datos. Por tanto, la latencia de envío de datos en la red es alta ya que los dispositivos R1, R2, R3, DF4, DF7 y DF8 (ver Fig. 3) están tratando de enviarle datos al coordinador ZigBee durante el mismo periodo de supertramas puesto que pertenecen a la misma PAN (a nivel de acceso al medio). Como se puede observar en la Fig. 9 hay ocho dispositivos finales enviándole datos al coordinador a una tasa constante de un paquete por segundo; o sea, que el coordinador debería estar recibiendo ocho paquetes por segundo, aunque en realidad está recibiendo aproximadamente 2,5 paquetes por segundo. En otras palabras, se están perdiendo más o menos 5,5 paquetes por segundo; además, la latencia de los paquetes dentro de la red es más o menos de 7,5 segundos (muy alta) [3].

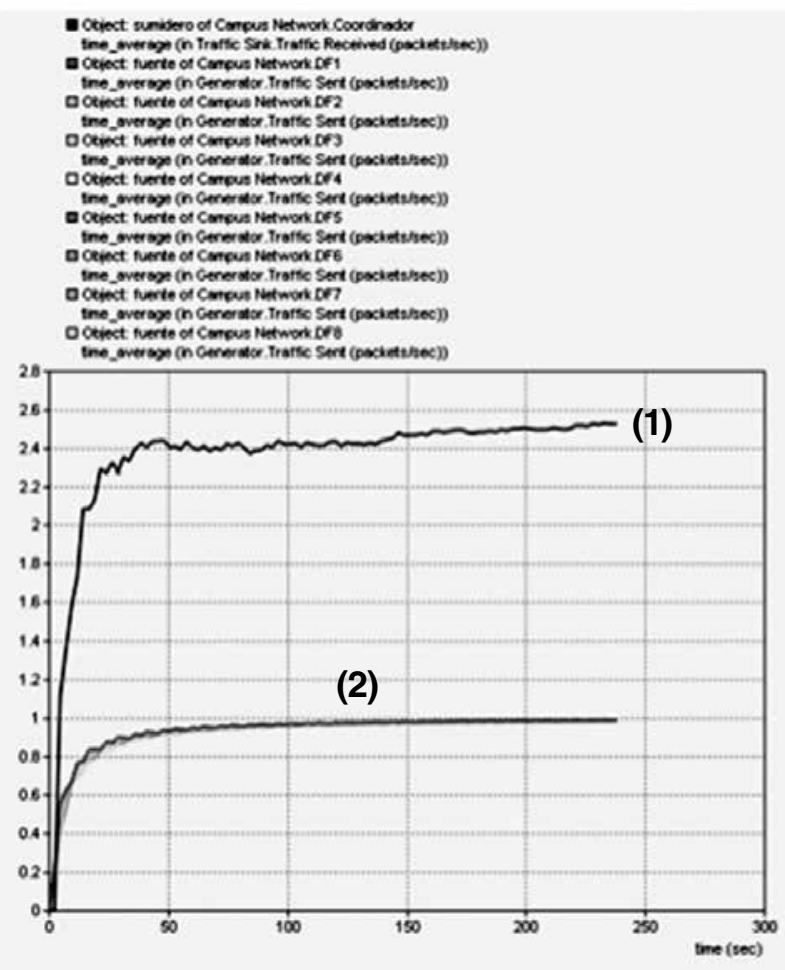

Fig. 9 Tráfico promedio de recepción de paquetes por parte del coordinador y tráfico promedio de envío de paquetes por parte de los dispositivos finales para la topología de red de la Fig. 3 [3] 
La solución para que se pierdan menos paquetes por el camino es incrementar el orden de la supertrama, o disminuir el orden de la beacon de los dispositivos que emiten beacon (coordinador ZigBee y Routers), pero sin llegar a afectar la calendarización de los periodos de duración de las supertramas (que los periodos de las supertramas en ningún momento se sobrepongan en el tiempo). Con lo anteriormente mencionado se mejoraría la latencia de los paquetes que son enviados dentro de la red [3].

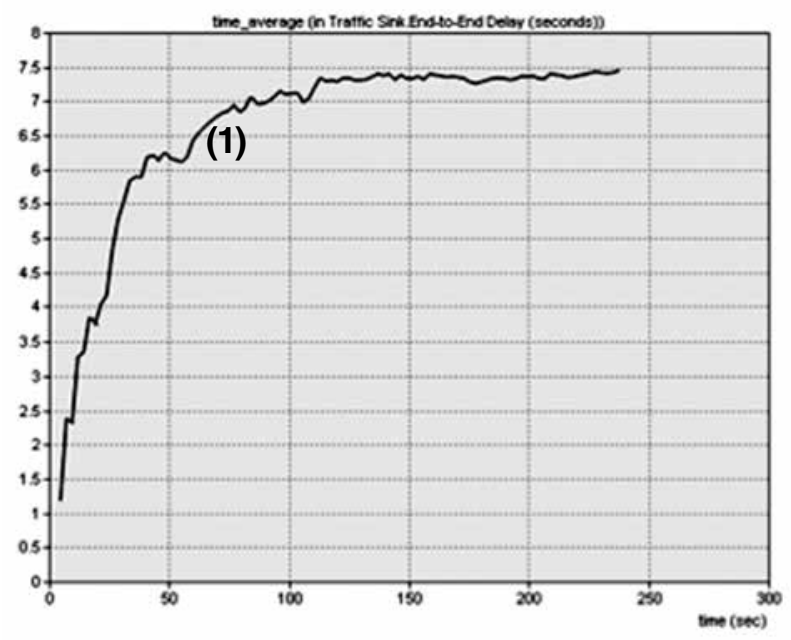

Fig. 10 Retardo sufrido por los paquetes de la topología de red de la Fig. 3 [3]

\section{Conclusiones}

En este trabajo se desarrolló un modelo de simulación de una WSN que utilizó las especificaciones IEEE 802.15.4/ZigBee [6], [4] (flujo de datos) más las especificaciones del Transductor CC 2420 [9] (consumo de energía). El modelo de simulación utiliza la configuración de sincronización periódica de la beacon habilitada (a nivel de subcapa MAC); esta configuración permite hacer ajustes a la supertrama en cuanto a duración e intervalo de ocurrencia, lo cual repercute en aspectos, tales como, el consumo de energía y latencia de los datos. Estos parámetros de consumo de energía y de latencia de los datos, se deben tener en cuenta a la hora de diseñar la red (para optimizarlos), dependiendo de la aplicación que se le quiera dar a esta [3].

Dentro del modelo de simulación se logró modelar el flujo de datos que hay entre cada una de las capas, gracias a que las especificaciones IEEE 802.15.4/ZigBee [6], [4] describen de forma clara el formato y función de los mensajes que deben ser utilizados para la comunicación entre capas. Esta comunicación entre capas, permite que las capas inferiores le puedan proveer servicios a las capas superiores (i.e. servicio de datos y servicio de gestión) [3].

El consumo de energía de una red de sensores inalámbricos es importante, ya que de este depende el tiempo de vida de la red. Las especificaciones del transductor CC2420 [9] son muy claras y permitieron determinar el consumo de energía en cada estado, lo que hizo posible modelar el consumo de energía de un nodo-sensor que tenga este transductor (modelo lineal), con lo cual, se puede estimar el tiempo de vida de un nodosensor [3].

Por medio de la simulación se logró comprobar que efectivamente el consumo de energía a nivel de nodo sensor no depende de la cantidad de datos trasmitidos o recibidos por este, sino que depende del estado (encendido o apagado) en que se encuentre el transmisor y/o receptor del nodo sensor (teniendo en cuenta que el trasmisor y el receptor no pueden estar encendidos al mismo tiempo [6]). El receptor del nodo sensor va a consumir la misma cantidad de energía si se encuentra encendido, independientemente de, si este está o no recibiendo datos; lo mismo ocurre con el transmisor [9] (si este está o no transmitiendo datos [consumirá la misma cantidad de energía]) [3].

Teniendo en cuenta los datos (gráficas) de la simulación, se concluyó que los nodos sensores de tipo router gastan mucha más energía (más o menos el doble [en este modelo lineal]) que los 
nodos sensores de tipo coordinador ZigBee o dispositivo final, debido al doble papel que deben cumplir los nodo sensores de tipo router (dentro de la red ZigBee [4]) para poder enrutar los datos, ya que durante un periodo de supertrama deben comportarse como dispositivos asociados a un coordinador PAN (están activos durante este periodo de tiempo), y durante otro periodo de tiempo deben comportarse como coordinadores PAN (también están activos durante este periodo de tiempo). De lo anterior se concluye que durante un intervalo específico de tiempo (intervalo de la beacon del coordinador ZigBee [comienzo de una trama beacon al comienzo de la siguiente trama beacon del coordinador ZigBee]), el coordinador ZigBee y los dispositivos finales solo estarán activos durante una duración de supertrama (en periodos diferentes), mientras que el router estará activo durante dos periodos de supertrama: una duración de supertrama cuando actúa como dispositivo asociado a un coordinador PAN, y otra duración de supertrama cuando actúa como coordinador de una PAN (para PANs diferentes, durante periodos de tiempo diferentes) [3].

Por medio de la simulación se encontró que efectivamente para una transmisión en la banda de $2450 \mathrm{MHZ}$, la mejor configuración de orden de la supertrama y orden de la beacon es de 0 y 6 respectivamente, tal como se recomienda en la especificación ZigBee [4], ya que se optimizan dos aspectos importantes de la red ZigBee [4]: el primero, que esta configuración hace que el tiempo de vida de un nodo sensor sea alto, lo cual es importante para la relación costo beneficio; lo segundo, que esta configuración permite la calendarización para las supertramas del coordinador Zigbee y los dispositivos de enrutamiento (la calendarización de las supertramas permite el enrutamiento dentro de la red) [3].

Por último, se concluye que el modelo de simulación se puede ampliar (para trabajos futuros) de varias formas. Una forma sería adicionándole servicios y funcionalidades que el modelo actual no posee (el modelo funciona con los requerimientos mínimos); otra forma sería calibrando el modelo, o sea dándole mayor precisión al modelo actual; otra, sería cambiar el modelo lineal de consumo de energía que se tiene, por un modelo que se acerque más a la realidad (e.g. modelo electroquímico o modelo estocástico de una batería) y otra forma sería utilizar el modelo que se desarrolló de la capa física y la subcapa MAC de la norma IEEE 802.15.4 [6], para probar otros protocolos de enrutamiento [3].

Para los trabajos futuros que quieran basarse en este modelo de simulación (ampliar y/o mejorar el modelo), se recomienda utilizar la metodología de la Fig. 5.

\section{REFERENCIAS}

[1] R. Nagarajan and R. Dhanasekaran, "Implementation of wireless data transmission in monitoring and control," Communications and Signal Processing (ICCSP), 2013 International Conference on, Melmaruvathur, 2013, pp. 83-87.

[2] T. Nakanishi, "A generative wireless sensor network framework for agricultural use," Electrical Engineering and Informatics (MICEEI), 2014 Makassar International Conference on, Makassar, 2014, pp. 205-211.

[3] M. O. Amarillo, "Simulación de redes de sensores inalámbricos: un modelo energético a nivel de nodosensor bajo las especificaciones IEEE 802.15.4TMY ZIGBEE", M.S. Thesis, Dept. Ing. Elect., Univ. Javeriana, Bog. D.C., 2012.

[4] ZigBee Specification, ZigBee Document 053474r17, January 17, 2008.

[5] A. Cunha et al., "Implementation Details of the Time Division Beacon Scheduling Approach for ZigBee Cluster-Tree Networks", isep, Portugal, Porto, Tech. Rep. TR070102, Jul.20, 2007. Disponible en http://hdl. handle.net/10400.22/3940

[6] IEEE Standard for Information Technology - Telecommunications and Information Exchange Between Systems - Local and Metropolitan Area Networks Specific Requirements Part 15.4: Wireless Medium Access Control (MAC) and Physical Layer (PHY) Specifications for Low-Rate Wireless Personal Area Networks (LR-WPANs), in IEEE Std 802.15.4-2003, vol., no., pp.0_1-670, 2003.

[7] TELOSB, MEMSIC Inc. Disponible en http://www. memsic.com/userfiles/files/Datasheets/WSN/telosb_ datasheet.pdf 
[8] MicaZ, MEMSIC Inc. Disponible en http://www.memsic.com/userfiles/files/Datasheets/WSN/micaz_datasheet-t.pdf

[9] CC2420, 2.4 GHz IEEE 802.15.4 / ZigBee-ready RF Transceiver, Chipcon Products from Texas Instruments.

[10] OPNET Modeler, OPNET University Program: disponible en http://www.opnet.com/university_program/ itguru_academic_edition/

[11] R. G. Sargent, "Verification and validation of simulation models," 2008 Winter Simulation Conference, Austin, TX, 2008, pp. 157-169. 